\title{
GLAD!
}

Revue sur le langage, le genre, les sexualités

$01 \mid 2016$

Varia

\section{La protection maternelle}

Catégorisations et hiérarchisations du féminin dans les discours juridiques d'après-guerre

Maternal Protection.How Post-War Judicial Discourses Categorise and

Hierarchise Femininity

\section{Anne-Laure Garcia}

\section{OpenEdition}

Journals

Édition électronique

URL : http://journals.openedition.org/glad/236

DOI : $10.4000 /$ glad.236

ISSN : 2551-0819

Éditeur

Association GSL

Référence électronique

Anne-Laure Garcia, « La protection maternelle », GLAD! [En ligne], 01 | 2016, mis en ligne le 15

novembre 2016, consulté le 17 décembre 2020. URL : http://journals.openedition.org/glad/236 ; DOI https://doi.org/10.4000/glad.236

Ce document a été généré automatiquement le 17 décembre 2020.

\section{c) (i) $९$}

La revue GLAD! est mise à disposition selon les termes de la Licence Creative Commons Attribution Pas d'Utilisation Commerciale - Pas de Modification 4.0 International. 


\title{
La protection maternelle
}

\author{
Catégorisations et hiérarchisations du féminin dans les discours \\ juridiques d'après-guerre
Maternal Protection.How Post-War Judicial Discourses Categorise and
Hierarchise Femininity

Anne-Laure Garcia

\section{Introduction}

1 La notion de protection porte en elle une différenciation entre le protecteur et le protégé. Cette opposition structurante illustre le caractère binaire et hiérarchique des schèmes de perception et d'appréciation. Une frontière est tracée entre deux catégories qui se voient attribuées une valeur inégale au vu des oppositions latentes qui y sont liées : le fort/ le faible, le défenseur/ le défendu, le bienfaiteur/ le bénéficiaire, etc. ${ }^{1}$ Le droit est un des principaux éléments participant à la légitimation des catégories ${ }^{2}$ protecteur et protégé ainsi qu'à la définition des risques ou dangers justifiant une mise à l'abri de certains agents sociaux. Lorsque l'appartenance à une catégorie de genre est ancrée dans le titre d'un texte juridique ayant trait à la protection, il semble que ce soient toujours les femmes qui en soient l'objet. La hiérarchie entre le féminin et le masculin que cela implique est, qui plus est, bien souvent renforcée par la juxtaposition à des catégories hautement dominées telles que les enfants ${ }^{3}$ ou les esclaves ${ }^{4}$.

2 Au-delà de la violence symbolique ${ }^{5}$ impliquée par de telles différenciations, il semble intéressant de se plonger plus profondément au sein de la catégorie des protégées afin d'observer les catégorisations et les hiérarchisations du féminin véhiculées par les discours juridiques. Pour ce faire, les textes sur la protection maternelle sont des matériaux particulièrement adéquats. En effet, ils fondent leur objet sur des différenciations entre les femmes. Le présent article propose donc de se fonder sur trois études de cas afin de mettre au jour les hiérarchies genrées portées par les figures féminines, ancrées dans des discours juridiques partageant une vision commune où les femmes en situation de parentalité sont soumises à des risques pouvant légitimer une 
action publique. Plus précisément, pour chacun des trois cas étudiés, nous nous demanderons : Quels dangers sont consacrés par les textes? Comment y sont distribués les rôles de protecteurs et de protégées? Quelles représentations de la normalité et de la déviance maternelle sont ancrées dans ces codifications? Quelles hiérarchisations entre les différentes catégories de mères, et plus généralement de femmes, y sont observables?

3 Afin d'accéder aux visions et divisions portées par le droit, il est nécessaire de développer une stratégie de recherche susceptible de mettre au jour les impensés incorporés. Celle-ci doit, premièrement, permettre une Verfremdung ${ }^{6}$ - condition préalable à un éclairage réflexif de ce qui semble «aller de soi ». Il faut, deuxièmement, que les catégorisations et hiérarchisations portées dans le droit soient considérées dans leur historicité. ${ }^{7}$ Troisièmement, une attention particulière doit être portée au langage utilisé dans les textes juridiques pour être en mesure de dévoiler les éléments non manifestes du discours juridique. Au vu de ces principes méthodologiques, cet article propose de mêler internationalisation, historicisation et analyse de contenu via trois études de cas.

4 Notre analyse se focalisera sur les textes législatifs traitant de la protection maternelle adoptés au sortir de la Seconde Guerre mondiale en France, en RFA et en RDA. À chaque fois, le propos sera structuré en deux temps. Tout d'abord, nous nous appuierons sur l'état de la recherche socio-historique ${ }^{9}$ afin de mettre au jour les phénomènes de dépendance au chemin emprunté (path depedence) dans lesquels sont ancrés les textes juridiques adoptés dans l'après-guerre. Puis, les résultats des trois analyses de contenu menées sur les textes adoptés entre 1945 et 1952 seront présentés. Ces trois études de cas successives seront, au sein de la conclusion, suivies d'une mise en regard posant les bases d'une réflexion plus transversale quant aux représentations du féminin véhiculées par les discours juridiques sur la protection maternelle.

\section{La protection assurantielle des mères actives ouest-allemandes}

5 La figure des Trümmerfrauen ${ }^{10}$ est un symbole bien connu d'une Allemagne vaincue où la survie se joue entre les décombres et sans les hommes. Ce tableau d'un Land der Frauen (Strecker 1981: 53), d'un pays où les femmes tiennent à bout de bras ce qui reste de la société, est consécutif à l'enrôlement de la quasi-totalité de la population masculine, puis à la lenteur voire à l'impossibilité de son retour. Même lorsque le déséquilibre numérique entre les sexes tend à reculer suite à des libérations, les femmes se retrouvent fréquemment seules à gérer le quotidien de leur foyer. Bien souvent, les couples implosent car les conjoints si attendus se révèlent être des inconnus qui sont physiquement amoindris et psychologiquement brisés. De manière temporaire ou définitive, de nombreuses mères allemandes sont donc amenées à exercer des fonctions sociales productives et reproductives. C'est dans ce contexte qu'est préparée puis adoptée la Loi fondamentale de la République Fédérale d'Allemagne en 1949. Ce texte ancre constitutionnellement le principe d'égalité entre les hommes et les femmes (art. 3) tout comme la différenciation des droits selon le sexe en cas de parentalité. En effet, l'article 6 - entièrement dédié aux questions familiales - distingue deux catégories de protection publique. Tout d'abord, l'alinéa 1 stipule que « le mariage et la famille sont sous la protection particulière de l'ordre étatique $»^{11}$. Cela signifie une 
interdiction constitutionnelle d'atteinte au couple légitime et aux cellules familiales impliquant des enfants. À côté de cette mission générale de consolidation publique de la sphère privée, l'alinéa 4 précise, ensuite, que «toutes les mères ont droit à la protection et l'assistance de la communauté ». Le binôme protection/assistance allié à l'idée d'un dû collectif présuppose que les ascendants féminins au premier degré ont des devoirs particuliers et que l'exercice des fonctions maternelles de soin et d'éducation (art. 6, al.2) est reconnu par la société. Ainsi, la Loi fondamentale précédant et conditionnant toute nouvelle loi recèle-t-elle le principe d'une égalité non mécanique entre les hommes et les femmes. Elle enracine et légitime l'idée que l'équité démocratique implique une modulation des droits et des devoirs en fonction du sexe du parent.

\subsection{Une couverture des travailleuses en couches héritée de l'Empire}

La loi fédérale de 1952 sur la protection maternelle est soumise au poids d'un héritage historique qui trace une voie dans laquelle elle est amenée à marcher. En effet, le terme Mutterschutz, dont l'utilisation politique est observable dès la fin du dix-neuvième siècle, est profondément enraciné dans une perspective d'assurance sociale. Deux principales catégories d'acteurs œuvrent alors en faveur d'une publicisation des risques sociaux liés aux couches en utilisant le concept de Mutterschutz: des personnalités issues du monde médical et des mouvements féministes (Garcia \& Wobbe 2011). Le discours sanitaire apparaît à la fin du dix-huitième siècle. Ainsi, le docteur Johann Peter Frank plaida-t-il dès 1784 en faveur d'une Polizey ${ }^{12}$ qui aiderait les femmes enceintes et qui organiserait les soins périnataux, tous deux étant pour lui des missions publiques (Frank 1784). À sa suite, en 1802, le gynécologue Franz Anton Mai proposa un premier projet de loi sur la protection maternelle qui prévoyait notamment un congé de quatorze à seize semaines pour les couches (Anton Mai 1802). Si, dans un premier temps, ces discours semblèrent tomber dans l'oubli, ils reprirent de la vigueur suite à l'édiction des premières législations prussiennes sur le droit du travail et la protection de l'enfance. La légitimation d'une mission publique de protection des mères semble donc dériver doublement de celle des enfants. En effet, non seulement les femmes constituent une seconde catégorie se différenciant de la norme du travailleur masculin adulte, mais elles servent aussi de maillon dans la mécanique pour sauvegarder les citoyens de demain. Par là même, un premier contenu cognitif est imprimé sur la notion de Mutterschutz: il s'agit de protéger les travailleuses face aux risques liés aux couches. À partir des années 1860 , les associations de femmes prolétaires qui luttent pour l'amélioration des conditions d'existence des ouvrières commencent elles aussi à utiliser cette notion lorsqu'elles promeuvent l'idée d'une assurance maternelle. À la fin du dix-neuvième siècle, les mouvements féministes de toutes obédiences soutiennent l'entrée dans le débat public de la question de l'arrêt de travail lié aux couches et de la sécurisation financière des mères (Hauser 2004: 73). La création, en 1905, d'une association féministe ancrant la protection maternelle au cœur même de son nom - der Bund für Mutterschutz $z^{13}$ - est symptomatique de l'établissement de cette notion au sein $\mathrm{du}$ vocabulaire politique allemand. La compréhension assurantielle de la protection maternelle est ainsi partagée par l'ensemble des courants politiques lorsque, à partir de 1878 , des règles protégeant la femme active en couches ${ }^{14}$ sont peu à peu adoptées. ${ }^{15}$

7 Cet héritage ancien tend à expliquer que le texte du 24 janvier 1952 relève exclusivement du droit du travail. La loi de protection des mères actives ${ }^{16}$ concerne donc 
uniquement les femmes enceintes et les jeunes mères occupant un emploi déclaré ${ }^{17}$. Ses domaines d'application sont, à ce niveau, assez larges : elle octroie le droit à un congé de couches; elle régule les possibilités d'heures supplémentaires, de travail la nuit et le dimanche, de pauses à des fins d'allaitement; elle protège les futures et jeunes mères contre les risques de licenciement et de perte de revenu ; elle interdit des activités qui pourraient aller à l'encontre du bon déroulement de la grossesse ou mettraient en danger la santé de l'enfant à naître ${ }^{18}$, etc.

\subsection{Une hiérarchisation des ayantes droit selon le degré de reproductivité de l'emploi}

8 La loi de 1952 distingue dans son chapitre préliminaire quatre catégories de travailleuses, et ce selon deux axes. Dans un premier temps, l'article premier définit les bénéficiaires de cette mesure. Il s'agit de toutes les «femmes ayant un contrat de travail » ainsi que des «employées féminines travaillant à domicile [...] dans la mesure où elles travaillent de façon continue ». Ainsi, le texte crée-t-il deux catégories de femmes actives en traçant la frontière au niveau du positionnement de l'emploi à l'intérieur ou bien à l'extérieur de l'espace privé. L'article suivant effectue, dans la foulée, un second travail de définition dont la portée normative n'apparaît pas immédiatement, mais qui sera structurante pour les dispositions à venir. La catégorie "employée de maison» (Hausgehilfin) correspond ainsi aux «femmes qui sont employées dans un foyer afin d'effectuer des travaux ménagers et qui vivent dans la communauté familiale de leur employeur» (art.2, al.1). La catégorie "assistante ménagère " (Tagesmädchen) ${ }^{19}$, quant à elle, se réfère aux « femmes qui, sans vivre dans la communauté familiale de leur employeur, sont employées de façon continue au sein du même foyer afin d'effectuer des travaux ménagers qui exigent l'intégralité de leur force de travail » (art. 2, al. 2). Ici, la localisation de l'emploi se fait au sein du domicile de l'employeur et la frontière est délimitée par le lieu d'habitation de la femme - soit dans ce «foyer d'accueil», soit dans son propre foyer. Le point commun entre ces deux catégories est que la fonction productive exercée par la salariée correspond aux fonctions reproductives traditionnellement réalisées gracieusement par les femmes du foyer.

Ces différenciations, bien loin d'avoir un simple objectif de clarification lexicale, portent en elles une hiérarchisation normative qui s'illustre tout au long du texte législatif. On peut en effet observer de nombreuses modulations des droits en fonction de l'appartenance à ces diverses catégories. En ce qui concerne l'allaitement d'un nouveau-né, l'alinéa 4 de l'article 7 adapte le droit de la mère salariée à une pause de 45 minutes toutes les 4,5 heures sans perte de salaire aux conditions particulières $d u$ travail à domicile. En effet, il exige que l'employeur rétribue $75 \%$ d'une heure de salaire en plus chaque jour afin de contrebalancer le temps passé à allaiter. Néanmoins, l'égalité entre les différentes catégories de mères actives s'arrête là. La protection face au licenciement au cours de la grossesse ainsi que pendant les quatre mois qui suivent l'accouchement ne vaut pas pour les employées de maison et les assistantes ménagères enceintes de plus de cinq mois (art.9, al.1). En découle l'article 11 qui octroie aux femmes licenciées l'assurance d'un revenu de remplacement équivalent à la moyenne des trois derniers salaires perçus, et ce jusqu'à ce que l'avancement de la grossesse leur donne accès aux indemnités liées au congé maternel. En ce qui concerne l'arrêt de travail avant la naissance de l'enfant, la hiérarchie entre les actives travaillant pour 
une entreprise - que ce soit au sein de ses locaux ou à domicile - et celles exerçant des fonctions reproductives rémunérées au sein de foyers est grande: l'arrêt de six semaines avant l'accouchement dont bénéficient les premières est réduit à quatre pour les secondes (art. 3, al. 2). Cet écart d'un tiers du congé prénatal est symptomatique de la représentation de la femme portée par ce texte. En effet, dans l'Allemagne fédérale d'après-guerre, les fonctions d'ordre reproductif - même si elles sont réalisées de façon rémunérée - sont considérées comme un domaine d'activité qui s'adapterait particulièrement bien à l'état de grossesse (Edel 1993: 99). Cette idée explique aussi pourquoi les employées de maison et les assistantes ménagères ne sont pas concernées par l'interdiction de travail la nuit et le dimanche pour les femmes enceintes et les mères allaitantes (art. 8, al. 1). ${ }^{20}$

L'obligation constitutionnelle d'apporter "protection et assistance » à l'ensemble des mères trouve un terrain d'application pour le moins circonscrit en RFA. Entrée dans le langage courant, la notion de protection maternelle ancre l'idée que les pouvoirs publics ont le devoir de sécuriser financièrement l'existence des femmes actives non aptes à travailler en raison d'une grossesse ou de couches. Les femmes au foyer, protégées par les revenus de leur conjoint, ne sont donc naturellement pas éligibles à ce droit. Au-delà de cette première division entre les femmes qui nécessitent ou non une garantie de revenu, l'envergure du rempart public sauvegardant les travailleuses enceintes ou venant d'accoucher varie. Par exemple, l'évaluation des dangers induits par les différentes activités professionnelles évolue. Ainsi les catégories «employée de maison » et " assistante ménagère » ont-elles, au gré des réformes, disparues du texte. Reste encore une interdiction de travailler pour de très nombreuses femmes enceintes car l'exercice de leur métier est considéré comme dangereux pour leur santé ou celle de leur enfant à naître. Les risques présumés liés au port régulier de charges dont le poids est supérieur à cinq kilogrammes et le maintien d'une posture debout impliquent respectivement de larges restrictions dès la déclaration de grossesse, puis une interdiction d'exercice à partir du cinquième mois de gestation pour de très nombreux groupes professionnels. Cela concerne notamment les ouvrières à la chaîne, les éducatrices de jeunes enfants, les aides-soignantes, les kinésithérapeutes ou bien les chirurgiennes. Ainsi la hiérarchisation des droits à la protection maternelle reste-t-elle fonction d'une vision où le corps de la femme nécessite une intervention externe afin de le sauvegarder.

\section{La garantie socialiste de l'émancipation maternelle}

11 En RDA, la conception engelsienne d'une émancipation féminine par le travail (Engels 2001 [1884]), alliée aux impératifs démographiques et économiques de l'après-guerre, fonde une triple identification constitutionnelle de la femme en tant que citoyenne, travailleuse et mère. Adoptée le 7 octobre 1949, la première Constitution est-allemande applique à la sphère privée le principe d'égalité des droits entre tous les citoyens (Garcia $2013:$ 62-65), et ce quel que soit leur genre : « Les hommes et les femmes sont égaux » (art. 7, al. 1), « Les lois et les dispositions qui portent atteinte à l'égalité entre les hommes et les femmes dans la famille sont abrogées» (art.30, al. 2), "Une naissance hors mariage ne peut impliquer de désavantages ni pour l'enfant ni pour ses parents» (art. 33). Néanmoins, l'exception au principe d'égalité est aussi constitutionnellement ancrée par l'édiction d'un principe de protection : «Le mariage 
et la famille sont au fondement de la vie de la communauté. Ils sont sous la protection de l'État » (art. 30, al. 1), "Pendant la maternité, la femme a droit à une protection particulière et à la sollicitude de l'État » (art. 32, al. 1). Ici, la Constitution prévoit même la promulgation d'une loi ainsi que son implémentation: «La République promulgue une loi de protection maternelle. Des établissements visant la protection de la mère et de l'enfant doivent être créées » (art. 32, al. 2). Deux processus de catégorisation sont là observables : l'un à l'aune du sexe, l'autre de la parentalité féminine à laquelle aucune borne temporelle n'est attribuée ${ }^{21}$.

\subsection{Veiller sur les mères soviétiques afin de mieux les surveiller}

12 Au-delà de l'héritage des législations prussiennes érigeant un système d'assurance sociale pour prévenir les risques liés à la grossesse et l'enfantement des femmes actives, le nouvel État socialiste est avant tout sous l'influence du modèle soviétique. Le premier décret russe en matière de protection maternelle et infantile fut promulgué dès le 19 décembre 1917. Les dirigeants appellent «les citoyennes, les mères, les spécialistes médicaux à lutter pour la sauvegarde des générations à venir " (OMS 1962 : 9). Les pouvoirs publics accordent un rôle actif à la femme en ce qui concerne la préservation de son enfant. Ainsi, la protection sociale des mères signifie-t-elle la garantie universelle d'un ensemble de droits et d'aides permettant d'assurer le bienêtre de la progéniture des femmes: sécurité de l'emploi, octroi d'allocations de naissance, versement du salaire complet pendant les 112 jours de congé de maternité, subvention à partir de la troisième naissance, etc. (id. : 17-18). Le volet sanitaire de la protection maternelle et infantile soviétique apporte, quant à lui, une composante d'un ordre nouveau à cette mission publique : l'éducation des mères. Au cours des huit consultations prénatales et des dix-huit consultations obligatoires jusqu'aux trois ans des enfants (ibid. : 19, 32), le corps médical doit éduquer les femmes et s'assurer de la qualité des soins apportés aux enfants. L'éducation publique aux fonctions maternelles et le contrôle de son bon apprentissage entrent, au moins à deux reprises, dans la sphère particulièrement intime du domicile familial ${ }^{22}$. Devenue ainsi permanente et obligatoire, la protection sanitaire équivaut désormais à un contrôle médical. La figure de la mère n'est plus celle d'une ayante droit bénéficiant de garanties publiques, mais d'une citoyenne dont le devoir est de mener à bien les fonctions reproductrices pour lesquelles elle a été éduquée. Elle n'est plus en danger : elle est le danger. De ce fait, la surveillance et l'évaluation publiques des comportements maternels servent avant tout à la protection de l'enfant contre sa mère, pensée comme potentiellement porteuse de risques.

13 Adoptée le 27 septembre 1950, la loi sur la protection maternelle et infantile et sur les droits de la femme ${ }^{23}$ se définit comme une réponse à la commande constitutionnelle estallemande (préambule, al.1). Son objectif explicite est de renforcer l'ordre social «antifasciste et démocratique » en permettant que l'égalité entre les hommes et les femmes soit non seulement un droit mais aussi un fait (préambule, al. 12). Pour ce faire, l'État garantit à toutes les citoyennes « une maternité heureuse » et des aides publiques afin d'" éduquer leur enfants dans un esprit de paix, de progrès et de démocratie " (préambule, al.3). Deux champs d'action principaux peuvent être identifiés: l'autonomie économique et la sécurisation sanitaire. Ainsi, afin de permettre la compatibilité des devoirs politiques, économiques et familiaux des citoyennes estallemandes, l'État garantit-il aux femmes qu'avoir un enfant à charge n'ira pas à 
l'encontre de leur autonomie économique. Pour une travailleuse, toute naissance donnera droit au paiement de cinquante marks et à un congé de onze semaines rémunéré à hauteur du revenu moyen des trois derniers mois (art. 10). Qui plus est, afin de permettre un emploi à temps plein des mères, 200000 places dans des institutions de garde collective avec de larges horaires d'ouverture ${ }^{24}$ sont prévues d'ici à 1955 (art. 5). Les employeurs ont, en outre, l'obligation de s'adapter aux particularités physiologiques des femmes (art.19) et aux obligations familiales des mères actives (art.23). Afin d'assurer la sécurisation sanitaire, la création de centres de conseil médico-sociaux (art. 6), de polycliniques, de services pédiatriques (art. 4) et de maisons de repos à destination des femmes enceintes à l'état de santé précaire (art.7) est prévue. Enfin, «dans l'intérêt de la protection de la santé de la femme et du soutien à une hausse des naissances", ce texte autorise l'interruption de grossesse uniquement pour des motifs d'ordre thérapeutique, et ce après approbation d'une commission d'experts (art. 11) ${ }^{25}$. La loi de protection maternelle est-allemande porte donc, tout comme sa cousine de l'Ouest, une vision où la sauvegarde du corps de la femme nécessite une intervention externe.

\subsection{Entre reconnaissance des mères en danger et suspicion d'un danger maternel} la protection maternelle: les familles nombreuses et les mères célibataires (al.3). Toutes deux ont en commun d'être désavantagées par rapport aux cellules familiales « normales » qui, en creux, sont définies comme un couple parental marié avec un à trois descendants directs. temps, pas pensée au niveau individuel. La protection publique dont elle peut bénéficier est partagée avec l'ensemble des membres de sa cellule familiale : «Des aides de l'État sont allouées en vue d'améliorer la situation matérielle des familles nombreuses et d'encourager le grand nombre d'enfants » (art. 1). Il semble donc que le problème public auquel la loi souhaite apporter une réponse soit le risque de pauvreté infantile induit par le fait que les revenus parentaux doivent couvrir les frais engendrés par les soins et l'éducation de davantage de personnes que la moyenne des ménages. Néanmoins, la déclinaison financière de ce principe de solidarité montre que le filet de sécurité est jeté en direction de la seule mère. Elle est en effet l'unique destinataire des primes attribuées à partir de la troisième naissance ${ }^{26}$ (art.2, al.1) ainsi que des allocations mensuelles à partir du quatrième enfant (art. 2, al. 2) ${ }^{27}$. L'attribution d'aides purement financières met en exergue que les reproductrices prolifiques doivent être protégées pour des motifs d'ordre strictement matériel. Qui plus est, l'augmentation progressive du montant des allocations attribuées à partir du cinquième enfant montre une reconnaissance accrue des fonctions reproductives des mères de familles très nombreuses. ${ }^{28}$

Il en est tout autrement des mères célibataires. La définition même de cette catégorie porte en elle une hiérarchie entre les femmes devenant mères au sein de l'institution du mariage et les autres. Les mères célibataires sont en effet celles «qui, de par la guerre ou pour d'autres raisons, ont été empêchées de se marier" (préambule, alinéa 4). Le verbe allemand « hindern » employé ici au passif signifie qu’une aspiration a 
été contrariée. Le souhait de la femme s'est donc heurté à un obstacle extérieur à sa volonté qui a entravé son accès à l'idéal du couple parental marié. Les dangers qui touchent ces femmes devraient donc être liés à leur monoparentalité supposée. Cette image de la mère célibataire comme victime - des aléas de la vie ou des hommes corrèle parfaitement avec les priorités pour l'attribution d'un logement et d'un emploi (art. 25) ainsi que pour l'octroi d'un mode de garde (art. 3, al. 3) que lui confère la loi. Néanmoins, le soutien à l'articulation des activités productives et reproductives prend, en cas de naissance hors mariage, une forme supplémentaire quelque peu particulière. La maternité célibataire est en effet mise en lien direct avec la possibilité de confier "son enfant » à un «foyer » impliquant une " prise en charge de l'ensemble des coûts par l'État » (art. 3. al. 1). Il y a ici trois présupposés. Tout d'abord, une femme seule n'a qu'un seul et unique enfant. Le célibat est donc non seulement synonyme de monoparentalité subie mais aussi d'abstinence - ou tout du moins de contrôle de la fécondité. Seule la mère non mariée est pensée comme pouvant se séparer de son enfant. La déviance face à la norme du couple parental semble donc suggérer que la maternité pourrait être non désirée ou bien non assumée. La loi protège ici non pas la mère mais l'enfant naturel sur qui plane le risque d'abandon. Enfin, la mère célibataire est suspectée de pouvoir vouloir tirer un avantage financier non justifié en cas de placement de l'enfant. Il est en effet spécifié que les « aides publiques pour l'enfant ne sont pas versées à la mère » ayant confié son enfant à un foyer (art. 3. al. 1). Ainsi la figure de la mère célibataire oscille-t-elle entre deux pôles : victime et coupable.

Dans le texte est-allemand de 1950 ici analysé, deux dangers fondent la protection des mères. Il s'agit tout d'abord des risques liés à des difficultés en matière de cumul des fonctions féminines. Être à la fois citoyenne, travailleuse et mère implique des stratégies de gestion du quotidien pouvant être simplifiées grâce à un réseau de structures publiques prenant en charge les enfants et à des dispositifs sécurisant les revenus des familles. Ainsi libérées des soucis matériels et assurées de pouvoir conserver leur autonomie, les mères protégées pourraient vivre une "maternité heureuse ». La seconde menace que la loi sur la protection maternelle est censée contrecarrer émane, quant à elle, des mères elles-mêmes. Cette méfiance justifie un apprentissage réglementé des soins aux nourrissons ainsi qu'un strict encadrement des risques de défection maternelle en cas de monoparentalité. La garantie universelle à une triple identité pour les Allemandes de l'Est associait donc féminité et maternité. Ce faisant, elle ancrait non seulement la norme de la compatibilité des fonctions productives et reproductives, mais aussi une dissymétrie entre les genres. Cette injonction soviétique au travail des mères ne fut pas sans provoquer des résistances dans la population allemande, ce qui mena au retour de la "journée du foyer» (Hausarbeitstag) ${ }^{29}$ puis à la mise en place d'une « année du bébé » (Babyjahr) rémunérées pour les mères actives. ${ }^{30}$

\section{La surveillance médico-sociale des mères en France}

L'organisation d'une politique nationale de protection maternelle et infantile fut, en France, réalisée dans l'immédiat après-guerre. L'acte de naissance de la PMI est en effet l'ordonnance $\mathrm{n}^{\circ}$ 45-2720 publiée par le gouvernement provisoire le 2 novembre 1945 , soit avant même l'édiction d'une nouvelle constitution démocratique. Une telle procédure est, d'un point de vue juridique, justifiée en cas d'extrême urgence afin de 
régler une situation ne pouvant souffrir une quelconque attente ou bien d'éviter qu'elle ne s'aggrave. Ce principe s'applique pleinement au texte édité par le gouvernement provisoire, aux yeux duquel il en va de la survie de la Nation: «La mortalité infantile atteint actuellement en France des chiffres si alarmants que des mesures rigoureuses doivent être prises immédiatement pour l'enrayer (...). C'est pour la France une question de vie ou de mort que de l'enrayer dans le plus bref délai » (exposé des motifs, al. 1 et 4$)$.

\subsection{L'universalisation du suivi sanitaire et social des mères françaises}

L'idée qu'une mortalité infantile excessive ferait courir au pays un risque vital remonte au dix-neuvième siècle. Premier territoire à faire l'expérience d'une transition démographique sans essor rapide de sa population, la France observe avec anxiété ses voisins rattraper voire dépasser le nombre de ses habitants. L'angoisse démographique, qui connut une croissance exponentielle suite à la défaite de 1870 et à l'union des États allemands en un Empire, conduisit des membres du monde médical à s'engager en faveur d'un suivi des grossesses et des couches. Le vénérologue Gustave Lagneau et l'obstétricien Adolphe Pinard ${ }^{31}$ comptent parmi les plus éminents professionnels de la santé œuvrant pour l'ouverture de refuges-ouvroirs accueillant les femmes enceintes indigentes et plaidant en faveur d'une loi qui institutionnaliserait un suivi fœtomaternel accessible à tous (Garcia 2013 : 44-47). C'est à ce carrefour entre craintes politico-démographiques et préoccupations médico-sociales que l'originalité de la compréhension hexagonale de la protection maternelle prend sa source. La spécificité réside, en effet, dans l'universalisation d'un dispositif sanitaire et social orienté vers une dyade mère-enfant. La protection maternelle est universelle car elle est conçue au sein d'une " politique élargie de protection de l'enfance " (Rollet 2008 : 193) dont elle est un élément parmi d'autres.

Si l'expression protection maternelle et infantile semble être apparue au cours de la Première Guerre mondiale (Hassani, Letoret, Morellec, 2009 : 34) afin de caractériser les projets médico-sociaux en faveur des jeunes enfants et de leurs mères, il est intéressant de noter qu' à partir de la fin du dix-neuvième siècle une autre notion est usitée dans les sphères politiques : la protection de la maternité. Cette appellation est observable dans les discussions sur la mise en place d'une assurance sociale régulant un congé lié à l'accouchement des femmes actives. Au-delà des projets développés par les parlementaires Albert de Mub, Fernand Engerand et Paul Strauss (Cova 1997), c'est ici la similarité avec le concept allemand de Mutterschutz qui attire l'attention. Il semble plausible que la notion de protection de la maternité - aujourd'hui disparue en France ait été importée d'outre-Rhin. En effet, si l'aïeul le plus ancien du congé de maternité est issu de Suisse, la loi fédérale sur les fabriques de 1877 ne traite de cette thématique que dans un seul alinéa et parle uniquement d'« un espace de temps » (art. 15, al. 2). Au même moment, dans l'Empire allemand, se préparait la loi sur la Mutterschutz qui fut adoptée en 1878. L'hypothèse d'une contamination temporaire du vocable allemand est, de plus, renforcée par le fait que les projets français se réfèrent à la conférence de Berlin de 1890 - une rencontre initiée par Guillaume II qui avait pour but de négocier une convention internationale sur le travail des enfants et des femmes dans les établissements industriels. 


\subsection{Les mères françaises : de bonnes et de mauvaises élèves} jeunes mères. Cependant, ce n'est pas le positionnement calendaire vis-à-vis d'une filiation qui structure la hiérarchisation parmi les mères à protéger. À partir du moment de la déclaration de grossesse, toutes les femmes font l'objet d'un suivi médico-social obligatoire ${ }^{32}$. La frontière qui sépare les ayantes droit est, en fait, liée à une distinction latente entre les bonnes et les mauvaises mères.

Les bonnes élèves, qui font preuve d'une coopération constante en matière d'apprentissage des savoirs et des pratiques se rattachant à la prophylaxie, à l'hygiène et aux soins des enfants en bas âge, bénéficient de dividendes méritocratiques : " Des primes d'assiduité seront accordées soit en espèces, soit en nature, aux futures mères qui fréquenteront régulièrement les consultations prénatales et les séances de vulgarisation organisées par ces consultations et au cours desquelles les intéressées acquièreront les premières notions de puériculture » (art. 12). Il en va de même pour les bonnes mères sans ressources qui suivent les recommandations en matière d'allaitement : elles perçoivent «une allocation supplémentaire pendant les six mois qui suivent l'accouchement » (art. 36). Cette récompense disparaît néanmoins dès que le comportement maternel dévie des règles dictées par la PMI : «Cette allocation n'est servie qu'à la condition formelle que la mère prenne, pour son enfant et pour ellemême, les soins d'hygiène visés par l'article 33 de la présente ordonnance » (art. 36).

Bien plus, les mauvaises mères se voient dépossédées du droit à la protection publique que leur accorde l'ordonnance. Ainsi, toute femme n'exerçant pas son "droit " à un suivi médical et/ou ne mettant pas à exécution les « conseils » qui lui sont « donnés » (art.11, al.1) encourt une "suspension partielle ou totale des allocations » (art.11, al.1). Il en va de même en cas d'urgence sociale. En effet, si «toute femme de nationalité française et privée de ressources suffisantes a droit, pendant la période de repos qui précède et qui suit immédiatement ses couches, à une allocation journalière " (art. 31, al. 1), ce filet de sécurité a priori universel n'est déployé qu'à condition que la bénéficiaire subisse tous les examens médicaux prescrits par l'ordonnance et qu'elle soit dans l'incapacité physique de travailler. L'ordonnance régule ainsi non seulement les récompenses mais aussi les punitions envers les mauvaises élèves. La mère n'est donc protégée que dans la mesure où elle ne grippe pas les rouages de la politique nataliste.

Notons ici que cette représentation dichotomique des mères fait écho à celle des deux figures professionnelles féminines citées dans l'ordonnance : l'assistante sociale et la nourrice. Il est intéressant de noter ici que l'on peut filer la métaphore scolaire afin de comprendre la hiérarchisation des différentes catégories de femmes censées prendre soin de l'enfant. La travailleuse gardant l'enfant d'autrui en échange d'une rémunération éveille clairement les soupçons de méconnaissance voire de malveillance. La gardienne qui ne respecte pas son devoir de déclaration préalable de son activité à la mairie s'expose à une amende voire à un emprisonnement pouvant durer jusqu'à six mois (art. 20 et 22). Ici, le vocabulaire juridique utilisé - « récidive » (art. 22), « délinquant» (art. 22), «infraction» (art. 25) - relève du droit pénal. La figure des « nourrices au sein » est, quant à elle, porteuse de risques spécifiques si bien que l'autorisation à exercer son métier est conditionnée à un contrôle sanitaire et administratif. ${ }^{33}$ Une telle précaution implique une forte défiance envers cette figure 
féminine qui, en se faisant rémunérer pour des comportements maternels, éveille les pires craintes en ce qui concerne le bien-être des enfants dont elle est censée prendre soin.

L'assistante sociale est, quant à elle, une inspectrice qui contrôle les pratiques sanitaires et éducatives des mères et des nourrices, puis qui en rend compte aux instances médicales et administratives. Le terme de "surveillance", cité six fois, implique une activité professionnelle d'observation, de contrôle puis d'évaluation des comportements familiaux. Ce suivi sanitaire et social est renforcé en cas de dangers avérés, c'est-à-dire si l'enfant est en nourrice, si les parents perçoivent des subsides publics en raison de difficultés matérielles, et si «les parents ont été condamnés pour mendicité ou ivresse à une peine correctionnelle " (art. 15, al. 2). Autrement dit, l'assistante sociale protège tout enfant victime de déviances sanitaires, matérielles ou morales de la part des adultes de sa famille. Elle n'est donc, pour les futures et jeunes mères, pas une protectrice mais une « surveillante générale ».

Si la représentation dichotomique de la mère - pouvant être tout aussi dangereuse que salvatrice pour son enfant - n'est pas nouvelle, l'ordonnance de 1945 enracine une surveillance universelle qui s'ajoute à la charité laïque héritée de la Révolution. Les rhétoriques de la prévention puis de promotion de la santé qui se sont par la suite développées n'ont en rien remis en cause le mélange entre surveillance et éducation qui fonde la notion de protection maternelle en France. Dans une perspective bourdieusienne, il semble qu'il s'agisse ici d'un prototype de violence symbolique, et plus précisément de domination symbolique. Subtile et quasiment invisible, la contrainte du contrôle ne devient intelligible qu'en cas de suspicion de déviance maternelle. Au-delà de l'appartenance à une catégorie considérée comme à haut risque $^{34}$ menant à une surveillance rapprochée prévue dès la déclaration de grossesse, toute femme peut potentiellement faire l'objet d'un contrôle inopiné à domicile si les normes édictées dans les carnets de grossesse et de santé du jeune enfant ne sont pas respectées. ${ }^{35}$

\section{Conclusion}

Si les motifs à l'origine d'une publicisation des risques liés à la maternité recoupent diverses polarisations et hiérarchisations structurantes quant à savoir ce que devrait être l'expérience féminine de la parentalité, un élément commun et fondamental se dessine dans les trois textes analysés : la mission publique de sauvegarde de l'enfant. Les droits consentis à la femme sont en effet au moins partiellement légitimés par l'idée d'une responsabilité collective à l'égard des tout-petits. Le droit maternel à une assistance, à une assurance sociale ou à un encadrement médico-social vise donc, dans les trois États, à préserver l'enfant des menaces qui pourraient mettre en péril son bon développement. Parfois, la mère devient alors une figure ambiguë vis-à-vis de sa progéniture qu'elle semble tout autant pouvoir sauver que mettre en péril.

L'analyse des discours juridiques sur la protection maternelle peut, au vu des résultats de cet article, servir de révélateur des normes genrées. La définition des risques et la hiérarchisation entre les différentes catégories d'actrices donnent, en effet, à voir les ascriptions $^{36}$ auxquelles est soumis le féminin. Pendant une période débutant avec la gestation d'un fotus et pouvant durer de quelques semaines à plusieurs années, leur sont attribués une responsabilité continue envers leur enfant, des risques de faiblesse 
physique, psychique et économique ainsi qu'une méconnaissance voire une malveillance qui légitiment que les pouvoirs publics veillent sur la dyade mère-enfant. Ce faisant, le droit sert également de pilier à la naturalisation de différences genrées socialement construites entre les femmes et les hommes en charge d'enfants, ces derniers ne justifiant pas une attention ciblée.

De ce fait, il semblerait légitime d'élargir la démarche mise en place au sein du présent article. Une analyse de la dimension genrée de l'articulation entre droit et sociétée ${ }^{37}$ centrée sur la notion protection serait ici particulièrement intéressante. Un travail se basant sur un large corpus de textes juridiques issus des droits nationaux et internationaux pourrait notamment permettre un regard plus systématique sur les interférences (Garcia 2013: 15) avec d'autres pans de l'action publique. En effet, des croisements et des influences réciproques semblent se dessiner dans les trois études de cas réalisées. Les injonctions à protéger les mères dans leur ensemble et/ou certaines catégories de mères pourraient, par exemple, être mises en regard avec les visées démographiques, les réformes en matière de droit de la famille, les politiques de l'emploi ou bien encore avec les objectifs affichés de maîtrise des dépenses publiques.

\section{BIBLIOGRAPHIE}

BOURDIEU, Pierre. 1986. « La force du droit. Éléments pour une sociologie du champ juridique » Actes de la Recherche en Sciences Sociales 64 : 3-19.

BOURDIEU, Pierre. 1998. La domination masculine. Paris : Seuil.

BOURDIEU, Pierre. 2001. Langage et pouvoir symbolique. Paris : Fayard.

BOURDIEU, Pierre. 2003. «L'objectivation participante » Actes de la recherche en sciences sociales $150: 43-58$.

CARDI, Coline \& DEVREUX, Anne-Marie. 2014. « Le genre et le droit : une coproduction. Introduction » Cahiers du Genre $57: 5-18$.

COVA, Anne. 1997. Maternité et droits des femmes en France. Anthropos : Paris.

EDEL, Ute. 1993. Die Entwicklung des Mutterschutzrechtes in Deutschland. Baden-Baden : Nomos Verlagsgesellschaft.

ENGELS, Friedrich. 2001 [1884]. Origine de la famille, de la propriété privée et de l'État. Paris : Broché.

FRANK, Johann Peter. 1784. System einer vollständigen medicinischen Polizey. Mannheim : C.F. Schwan.

GARCIA, Anne-Laure. 2013. Mères seules. Action publique et identité familiale. Rennes : PUR.

GARCIA, Anne-Laure \& WOBBE, Theresa. 2011. « Maternité, Mutterschaft, Mütterlichkeit : Familienpolitische Codierung im deutschen und französischen Kontext » in Die gesellschaftliche Verortung des Geschlechts. Diskurse der Differenz in der deutschen und französischen Soziologie 
um 1900, WOBBE, Theresa, BERREBI HOFFMANN, Isabelle \& LALLEMENT, Michel (éds.). Frankfurt am Main/New York : Campus, 114-141.

HASSANI, Anne, LETORET, Anne \& MORELLEC, Jeanne. 2009. Protection maternelle et infantile. De la promotion de la santé à la protection de l'enfance. Berger-Levrault : Paris.

HAUSER, Karin. 2004. Die Anfänge der Mutterschaftsversicherung : Deutschland und Schweiz im Vergleich. Zurich : Chronos.

LENOIR, Rémi. 2012. «L'État selon Pierre Bourdieu » Sociétés contemporaines 87 : 123-154.

LÉPINARD, Éléonore. 2006. «Faire la loi, faire le genre : conflits d'interprétations juridiques sur la parité » Droit et société 62 : 45-66.

MAI, Franz Anton. 1802. Der Polizei-Arzt am Gerichtshof der medizinischen Polizeigesetzgebung, Mannheim : Labisch.

MOELLER, Robert. 1997. Geschützte Mütter. Frauen und Familien in der westdeutschen

Nachkriegspolitik. Munich : Deutscher Taschenbuch Verlag.

ROLLET, Catherine. 2008. Les carnets de santé des enfants. La Dispute : Paris.

ORGANISATION MONDIALE DE LA SANTÉ [OMS]. 1962. La protection maternelle et infantile en URSS. Genève : Cahiers de Santé Publique.

STRECKER, Gabriele. 1981. Überleben ist nicht genug. Frauen 1945-1950. Freibourg : Herder Verlag.

\section{NOTES}

1. Selon Pierre Bourdieu, le fondement d'une telle structuration de la pensée au sein de deux classes complémentaires et hiérarchisées est le principe de division du monde entre le féminin et le masculin (1998).

2. Pierre Bourdieu parlait, à ce propos, de «la force du droit» (1986). Ce concept pose l'hypothèse d'un "pouvoir symbolique de nomination» (id.:13) des textes juridiques. Cette capacité performative repose tout d'abord sur la légitimation des divisions et visions du monde, c'est-à-dire des catégorisations et des représentations des catégories. Le processus de classification engendré par le droit est donc toujours double : la différenciation des catégories de pensée implique per se des hiérarchisations entre ces mêmes catégories. Le pouvoir symbolique nécessite, ensuite, un « effet doxique » expliquant que ces schèmes cognitifs ancrés dans le droit s'imposent aux agents sociaux sans même qu'ils en aient conscience. Une telle adhésion à des découpages évaluatifs du monde social correspond à une " proto-légitimité » (Lenoir 2012 : 135). Cette soumission immédiate relève, en effet, de la croyance consensuelle impliquée par un processus d'incorporation imbibant les structures cognitives des agents sociaux, bref des "présupposés impensés de la pensée pensante» (Bourdieu 2003 : 51). Ainsi les oppositions et hiérarchisations ancrées dans les textes juridiques structurent-elles les schèmes cognitifs fondant toute connaissance et reconnaissance du monde social.

3. C'est par exemple le cas dans la « Déclaration sur la protection des femmes et des enfants en période d'urgence et de conflit armé » adoptée par les Nations Unies en 1974.

4. Le décret du gouvernement français créant en 2013 la «Mission interministérielle pour la protection des femmes victimes de violences et la lutte contre la traite des humains » en est une illustration récente.

5. Cet outil théorique élaboré par Pierre Bourdieu met en exergue l'acceptation préréflexive des rapports de domination structurant l'ordre social. L'adhésion à la sociodicée fondant - voire 
naturalisant - les relations entre dominants et dominés est intrinsèquement liée à "l'imposition masquée (donc méconnue comme telle) de systèmes de classement et de structures mentales objectivement ajustées aux structures sociales » (Bourdieu $2001: 29$ ).

6. Cette notion, originellement formulée par Bertolt Brecht afin de décrire un effet théâtral, recouvre en sociologie l'idée d'une distanciation des évidences familières occasionnant un regard réflexif sur la réalité sociale.

7. La sociogenèse des lois est une étape indispensable dans l'effort de distanciation car elle permet d'observer comment une doxa s'est formée.

8. Au-delà de la quasi-simultanéité du travail de codification juridique, ce choix se justifie par la haute comparabilité entre ces cadres. Afin qu'une comparaison puisse mettre en lumière des similitudes et des différences sociologiquement pertinentes, qu'elle puisse permettre de mieux comprendre le réel, les unités mises en regards doivent faire preuve d'une certaine comparabilité. La France et l'Allemagne, deux pays géographiquement voisins, ont des caractéristiques structurelles communes offrant, d'un point de vue général, un cadre potentiellement fécond pour des études comparatives (morale judéo-chrétienne, appartenance à la famille de droit dite romano-germanique, quasi-simultanéité des développements économiques et sociaux, participation aux deux conflits mondiaux, etc.). Qui plus est, en matière d'action publique en direction des familles, la perspective franco-allemande peut se prévaloir d'être particulièrement intéressante, notamment du fait d'un regard réciproque sur ce qui a cours de l'autre côté du Rhin. D'un point de vue historique, par exemple, l'émergence et l'influence du courant d'idée nataliste en France ont été intrinsèquement liées à des préoccupations patriotiques et à la concurrence militaire avec les autres pays européens, notamment avec l'Allemagne. En effet, la défaite infligée au Second Empire par l'armée prussienne en 1870 a été essentielle pour le développement de cette conception envisageant la lutte contre le déclin des naissances comme un enjeu de survie nationale et développant une représentation de la famille en tant qu'affaire publique (Garcia 2013).

9. Quelques sources historiques ont été également mobilisées car leur importance était soulignée dans diverses publications socio-historiques.

10. Littéralement traduisible par «femmes des décombres», les Trümmerfrauen sont ces femmes qui, dans une Allemagne vidée de ses hommes valides, déblayèrent les gravats dus aux bombardements.

11. Tous les extraits de lois germanophones cités dans cet article ont été traduits par son auteure.

12. Tout comme son équivalent français police, le mot Polizey provient du mot latin politia, lui-

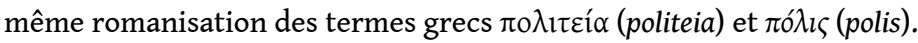

13. L'Alliance pour la Protection maternelle.

14. Ces mesures, liées à l'industrialisation, étaient principalement motivées par un souci d'amélioration des conditions de travail des ouvrières en couches et de protection des enfants à naître puis des nouveau-nés. Il s'agissait, par exemple de congés pré- et postnataux, d'indemnités d'allaitement, de l'interdiction de certaines activités, de la limitation des heures de travail, etc. (Edel : 21-67, Hauser : 25-63).

15. Sous le Troisième Reich, au nom de l'idéologie national-socialiste, cet héritage a été aryanisé par la loi du 17 mai 1942 qui intègre une condition de nationalité et de race (Edel 1993 : 83). Après la chute du régime, le Conseil de Contrôle en Allemagne abroge toutes les dispositions législatives motivées par le caractère discriminatoire de l'idéologie nationale-socialiste. Cela signifie que l'ensemble des travailleuses, quelles que soient leur nationalité ou leurs origines, accèdent aux protections établies en 1942. Il s'agit, par exemple, de l'interdiction du travail le dimanche, les jours fériés et de vingt heures à six heures pour les femmes enceintes et les mères qui allaitent ainsi que de la limitation du temps de travail à neuf heures par jour (Edel 1993: 83). Cette loi apportait à l'ensemble des femmes de l'Allemagne occupée deux protections particulièrement 
importantes: une limitation des possibilités de licenciement et l'assurance du paiement d'indemnités correspondant au salaire moyen des treize semaines d'activité précédant le congé de couches.

16. Gesetz zum Schutz der erwerbstätigen Mutter (aussi appelée Mutterschutzgesetz ou MuSchG).

17. À l'exception des fonctionnaires qui ont leurs propres règles.

18. Exposition au froid, à la chaleur, à la poussière, à la vapeur, à des radiations ou à des substances nocives ; port de lourdes charges ; longues phases debout, penchée ou accroupie, etc.

19. Cette traduction inspirée du terme "assistante maternelle» peut a priori paraître anachronique, mais elle se justifie par l'analogie avec le mot «Tagesmutter»- littéralement: « mère de jour » - qui signifie « nourrice ».

20. Notons ici que de nombreux secteurs d'activité voient ce droit être modulé en raison d'horaires et de jours de travail spécifiques (restauration, agriculture, monde du spectacle, etc.). Néanmoins, ces femmes bénéficient, contrairement aux employées de maison et aux assistantes ménagères, d'une pause d'au moins 24 heures un autre jour de la semaine (art. 8, al. 4).

21. Les droits auxquels accèdent les femmes via leur passage dans la catégorie mères disparaissent peu à peu, ceci en fonction de leur degré de responsabilité envers leur descendance. À titre d'exemple, les allocations familiales leur sont versées jusqu'à la fin de la scolarisation dans un établissement général.

22. Deux consultations médicales ont lieu à domicile. La première, réalisée par un pédiatre et une infirmière pendant le dernier mois de la grossesse, sert des fins éducatives et l'observation des "conditions socio-économiques dans lesquelles l'enfant naîtra (OMS 1962 : 19). La seconde visite de ces deux acteurs se déroule dans les trois jours suivant la sortie de la maternité et a pour "but essentiel (...) de poursuivre l'éducation de la mère et de s'assurer que les soins donnés à l'enfant, et particulièrement l'allaitement, sont réalisés correctement " (OMS $1962: 32$ ).

23. Gesetz über den Mütter- und Kinderschutzgesetz und die Rechte der Frau.

24. En général, de six heures du matin à dix-neuf heures, soit treize heures par jour.

25. L'objectif est donc de sauvegarder le fotus en cours de gestation et de préserver les capacités reproductrices de la femme, et non pas de garantir l'autodétermination des citoyennes. Cet article semble donc davantage mettre en lien avec l'objectif d'une protection infantile qu'avec celui d'une protection maternelle, voire des droits de la femme impliqués dans le titre de la loi.

26. $100 \mathrm{DM}$ pour le troisième enfant, $250 \mathrm{DM}$ pour le quatrième, $500 \mathrm{DM}$ pour tous ceux qui suivront.

27. $20 \mathrm{DM}$ pour le quatrième enfant puis $25 \mathrm{DM}$ pour les suivants, et ce jusqu'à leurs quatorze ans. 28. L'analyse du texte législatif ne permet pas de mettre en lumière les raisons de ce ciblage qui pourrait être lié à un risque de défection paternelle ou à une mise en lien normative de la mère et de ses descendants.

29. En 1939, le Troisième Reich avait introduit une "journée du linge » rémunérée pour les actives recensées comme « aryennes ».

30. Les pères ne pouvaient accéder à ces droits que si la mère et les grands-mères de ses enfants étaient dans l'incapacité avérée d'exercer ce droit.

31. À partir de 1919, Adolphe Pinard devint membre de l'Assemblée nationale.

32. Les presque deux-milles centres médico-sociaux relevant des services départementaux de PMI sont le pan le plus visible localement de ce suivi parce qu'ils sont implantés sur l'ensemble du territoire. Cependant, il est le moins utilisé car l'immense majorité des femmes consulte des professionnels de santé libéraux et hospitaliers qui sont, eux aussi, des acteurs à part entière du dispositif de protection maternelle et infantile.

33. Un médecin doit tout d'abord attester « qu'elle est apte à allaiter et n'est atteinte d'aucune maladie transmissible, qu'elle a subi les examens de dépistage de la syphilis et de la tuberculose » (art. 24, al. 1). Puis, le maire de sa commune de résidence doit indiquer par écrit « son état-civil » 
et fournir un extrait d'acte de naissance pour son dernier enfant afin que la preuve soit apportée qu'il est âgé de plus de six mois (dans la mesure où l'enfant est vivant).

34. Par exemple, une maternité précoce, l'absence de conjoint ou bien des difficultés financières.

35. Par exemple, si le calendrier de suivi n'est pas respecté ou bien si la mère et/ou l'enfant sont catégorisés comme en sous-poids par le corps médical.

36. Ce concept anglophone est difficilement traduisible en français. Il correspond aux attributions de caractéristiques en fonction de l'appartenance des acteurs à des catégories socialement construites.

37. Le droit «fait du genre » (Lépinard, $2006: 49)$ tout autant qu'il « est pétri de genre » (Cardi/ Devreux, $2014: 5$ )

\section{RÉSUMÉS}

La notion de protection porte en elle une différenciation entre le protecteur et le protégé. Cette opposition structurante illustre le caractère binaire et hiérarchique des schèmes de perception et d'appréciation. Afin de mettre au jour les rapports de genre portés par les discours juridiques sur la protection des femmes, cet article analyse les textes juridiques sur la protection maternelle adoptés entre 1945 et 1952 en France, en RFA et en RDA. Ces trois études de cas mettront en lumière les catégorisations et les hiérarchisations entre les figures féminines ancrées par ces discours juridiques.

The notion of protection implies a differentiation between the protector and the protégé. This structuring opposition illustrates the binary and hierarchical characteristic of schemes of perception and appreciation. In order to shed light on the gendered relations fixed by forensic discourses about protection of women, this article analyzes the law texts about protection of mothers, that were adopted between 1945 and 1952 in France, in the FRG and in the GDR. Those three case studies point up the categorizations and the hierarchizations between the female figures fixed by those juridical discourses.

\section{INDEX}

Thèmes : Recherches

Keywords : law, protection, mothers, categorization, hierarchization

Mots-clés : droit, protection, mères, catégorisation, hiérarchisation

\section{AUTEUR}

\section{ANNE-LAURE GARCIA}

Enseignante-chercheuse en sociologie, Chaire de microsociologie de l'Université de Dresde. Chercheuse associée au Centre Marc Bloch (Berlin). 